\title{
SNP identification and marker assay development for high-throughput selection of soybean cyst nematode resistance
}

\author{
Zi Shi ${ }^{1}$, Shiming Liư ${ }^{2}$, James Noe ${ }^{3}$, Prakash Arelli ${ }^{4}$, Khalid Meksem² ${ }^{2}$ and Zenglu $\mathrm{Li}^{{ }^{*}}$
}

\begin{abstract}
Background: Soybean cyst nematode (SCN) is the most economically devastating pathogen of soybean. Two resistance loci, Rhg 1 and Rhg4 primarily contribute resistance to SCN race 3 in soybean. Peking and PI 88788 are the two major sources of SCN resistance with Peking requiring both Rhg1 and Rhg4 alleles and PI 88788 only the Rhg 1 allele. Although simple sequence repeat (SSR) markers have been reported for both loci, they are linked markers and limited to be applied in breeding programs due to accuracy, throughput and cost of detection methods. The objectives of this study were to develop robust functional marker assays for high-throughput selection of SCN resistance and to differentiate the sources of resistance.

Results: Based on the genomic DNA sequences of 27 soybean lines with known SCN phenotypes, we have developed Kompetitive Allele Specific PCR (KASP) assays for two Single nucleotide polymorphisms (SNPs) from Glyma08g11490 for the selection of the Rhg4 resistance allele. Moreover, the genomic DNA of Glyma18g02590 at the Rhg 1 locus from 11 soybean lines and cDNA of Forrest, Essex, Williams 82 and PI 88788 were fully sequenced. Pairwise sequence alignment revealed seven SNPs/insertion/deletions (InDels), five in the 6th exon and two in the last exon. Using the same 27 soybean lines, we identified one SNP that can be used to select the Rhg1 resistance allele and another SNP that can be employed to differentiate Peking and PI 88788-type resistance. These SNP markers have been validated and a strong correlation was observed between the SNP genotypes and reactions to SCN race 3 using a panel of 153 soybean lines, as well as a bi-parental population, $F_{5}$-derived recombinant inbred lines (RILs) from G00-3213 x LG04-6000.

Conclusions: Three functional SNP markers (two for Rhgl locus and one for Rhg4 locus) were identified that could provide genotype information for the selection of SCN resistance and differentiate Peking from PI 88788 source for most germplasm lines. The robust KASP SNP marker assays were developed. In most contexts, use of one or two of these markers is sufficient for high-throughput marker-assisted selection of plants that will exhibit SCN resistance.
\end{abstract}

Keywords: Soybean cyst nematode, Resistance, SNP, KASP marker assays, Marker assisted selection

\section{Background}

Soybean cyst nematode (SCN, Heterodera glycines L.) is the most economically devastating pathogen of soybean and causes losses in soybean producing areas worldwide with no practical means of eradication [1]. The annual losses exceed one billion dollars in the United States alone [2]. Two resistance genes for SCN, Rhg1 (Resistance to H. glycines) on Chromosome (Chr) 18 and Rhg4.

\footnotetext{
* Correspondence: zli@uga.edu

${ }^{1}$ Center for Applied Genetic Technologies \& Dep. of Crop and Soil Sciences, University of Georgia, Athens, GA 30602, USA

Full list of author information is available at the end of the article
}

on Chr08, have been identified in the soybean germplasm, and have been shown to be the key sources of resistance in soybean cultivars [3]. The Rhg1 locus, which is crucial for broad spectrum resistance to $\mathrm{SCN}$, has been identified in various resistance sources, such as PI 88788 and Peking $[4,5]$.

SCN field populations are genetically variable [6,7]. Sixteen $\mathrm{SCN}$ races have been described and the race designation was determined by comparing its reproduction on a set of four soybean differentials with a standard SCNsusceptible cultivar. To avoid the implication of genetic predictability, a revised classification scheme for field SCN 
population introduced the Heterodera glycines (HG) types determined by reactions to seven indicator lines [7]. The distribution of SCN populations in the field varied among different geographic regions. However, race 3 is the most frequently found SCN populations in major soybean producing states in the USA [8,9]. Soybean lines PI 88788 and Peking are the two main sources of resistance to SCN race $3[10,11]$. Over $90 \%$ of the commercially available SCN-resistant soybean cultivars in the USA carry the rhg1$b$ allele, which is derived from PI 88788 due to its desirable agronomic characteristics [1,12,13]. While PI 88788-type resistance only requires the $R h g 1$ resistance allele, Pekingtype resistance needs both $R h g 1$ and $R h g 4$ resistance alleles for a resistant phenotype [14,15]. Although the molecular basis of SCN-resistance is still unknown, recent studies have shed light on the genes involved in the resistance. Map-based cloning showed that a single gene at Rhg4 locus encoding a serine hydroxymethyltransferase (SHMT, Glyma08g11490) is responsible for SCN resistance [14]. Two polymorphisms have been identified in the coding sequence of SHMT, $389 \mathrm{G} / \mathrm{C}$ in the first exon and 1,165 A/T in the second exon, which resulted in the amino acid change of Arginine ( $\mathrm{R})$ to Proline $(\mathrm{P})$ and Tyrosine $(\mathrm{Y})$ to Asparagine $(\mathrm{N})$, respectively. These polymorphisms change the property of the enzyme and may correlate with Rhg4-dependent resistance and susceptibility [14]. Genetic mapping and gene functional analysis of the rhg1-b locus exhibited a $31.2 \mathrm{~kb}$ genomic segment encoding a predicted amino acid transporter (Glyma18g02580), an $\alpha$-SNAP protein (Glyma18g02590) and a protein with wound-inducible protein 12 (WI12) (Glyma18g02610) [12,16]. Gene silencing and overexpression analysis showed that all three genes contribute to $\mathrm{SCN}$ resistance [12]. Interestingly, the phenotypic reaction to $\mathrm{SCN}$ is associated with the copy number variation of this $31.2 \mathrm{~kb}$ fragment. Only one copy is present in susceptible varieties, such as Williams 82 , and multiple copies in SCN resistant lines. For example, Peking possesses three copies and seven to 10 copies were detected in PI 88788derived resistant lines [12]. Sequencing of cDNA from several soybean lines revealed that multiple alleles of Glyma18g02590 were present in the different multi-copy Rhg1 categories [16].

Planting of resistant soybean cultivars combined with crop rotation formulates the best management strategy for reducing yield losses due to SCN. However, traditional breeding for resistant lines is challenging, not just because the phenotyping of SCN is time-consuming, but it is also affected by the different resistance genes [17] and varied SCN populations in the field [6,7]. Therefore, the use of molecular markers to assist the selection of SCN resistance is a crucial improvement in breeding programs. Major efforts have been made to develop genetic markers linked to SCN resistance genes. Restriction fragment length polymorphisms (RFLPs) markers have been identified and reported to facilitate soybean breeding for both Rhg1 [3,18-20] and Rhg4 loci [15,21-23]. Simple sequence repeats (SSRs) have further been discovered and applied in the marker-assisted selection of $\mathrm{SCN}$ resistance [15,21,22,24-27]. SNP/InDel markers have also been developed at the flanking region of both loci [11,28-30]. However, they are not diagnostic markers, and both SSRs and RFLPs are labor/time-consuming and cost ineffective. Thus, they are very limited to be applied in breeding programs in a high throughput setting.

Here, we report the development of three robust, functional SNP marker assays, two for the Rhg1 locus and one for $R h g 4$. These functional SNP markers provide genotype information which can be used for the high-throughput selection of SCN resistance, as well as differentiating Peking and PI 88788 resistance sources for breeding of SCN resistance cultivars and germplasm introgression.

\section{Results}

\section{SNP discovery}

To determine SNP markers within the Rhg1 locus, published sequences of the region were gathered which included the entire $31.2 \mathrm{~kb}$ block from PI 88788 and Williams 82 [12], but only the cDNA of Glyma18g02590 from Peking was available [31]. Moreover, we fully sequenced the genomic DNA of Glyma18g02590 from 11 soybean lines and cDNA of Glyma18g02590 from Forrest, Essex, Williams 82 and PI 88788 at the Rhg1 locus. Sequence alignment was only carried out for Glyma18g02580, Glyma18g02590 and Glyma18g02610, because other genes (partial Glyma18g02570 and Glyma18g02600) in this $31.2 \mathrm{~kb}$ block are not functional according to the previous study [12]. Three SNPs were identified in Glyma18g02580 from the comparison between Williams 82 and PI 88788, one synonymous SNP in the first exon, one in the intron and one in the 3' untranslated region (UTR) (Figure 1B). Glyma18g02590 possesses more SNP/InDels. The alignment of both published sequences (alleles in black) and our sequencing result of 11 soybean lines (alleles in red) revealed five SNPs in the 6th exon and two SNPs in the last exon. All of them lead to amino acid changes in the protein. Three SNPs from the 5'UTR of Glyma02610 have also been demonstrated between Williams 82 and PI 88788.

To evaluate which SNPs correlate with known SCN reactions of soybean lines, Kompetitive Allele Specific PCR (KASP) assays were developed and tested for all 13 SNPs at the Rhg1 locus and two previously identified SNPs (Figure 1A) at Rhg4 locus [14] using 27 soybean lines with known SCN reactions. Two SNPs from Glyma08g11490 are associated with the Rhg4 resistance allele, and they are completely linked (data not shown). Three SNPs in Glyma18g02580 and two SNP/InDels in the last exon of Glyma18g02590 are associated with the Rhg1 resistance allele, and all five markers are 

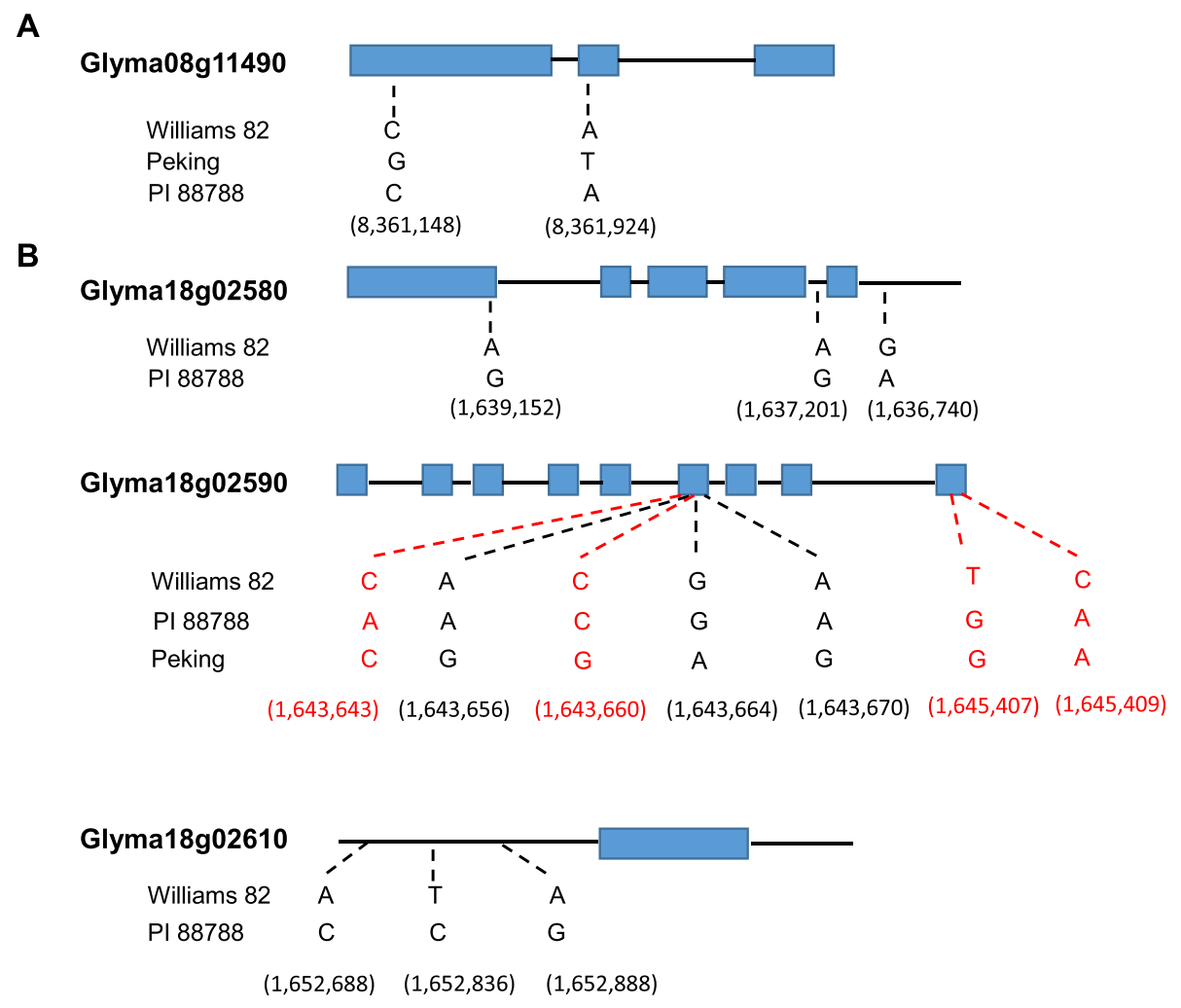

Figure 1 Schematic graph showed the position of SNP/InDels at Rhgl and Rhg4 loci. A. Two SNPs identified at Glyma08g11490 at Rhg4 locus. B. $13 \mathrm{SNP} / \mathrm{InDels}$ identified in three genes within a $31.2 \mathrm{~kb}$ block at Rhg1 locus. Blue squares represent exons and black lines represent UTRs and/ or introns. The genome coordinates (Database: Gmax_275_v2.0.softmasked) are shown under each SNP in parenthesis. SNP alleles in black are from published sequences and SNP alleles in red from our sequencing results.

completely linked. Five SNPs in the 6th exon of Glyma18g02590 could distinguish the sources of Pekingtype and PI88788-type resistance. However, three SNPs identified in the 5'-UTR of Glyma18g02610 showed no correlation with SCN resistance. Three SNPs were selected to provide genotype information for the selection of SCN resistance (Figure 2, Table 1), which were designated as GSM383, GSM381 and GSM191. GSM381 and GSM383 are located in the 6th and last exons of Glyma18g02590 at the Rhg1 locus, respectively, while GSM191 is in the first exon of Glyma08g11490. The KASP assays of three SNPs performed with 27 soybean lines exhibited a good clustering of the homozygous alleles, suggesting that it is feasible to employ those markers for selection.

\section{Haplotype comparison of resistance sources}

Haplotype analysis was performed using the Soy50k SNP Infinium chip data of 27 lines and nine cultivars including Woodruff, Hartwig, Boggs, Gordon, Bryan, G00-3880, Prichard, Bedford and Carver. The Rhg1 region was compared between Peking and the Peking-type lines: Forrest, Woodruff, Hartwig, Boggs, Gordon, Bryan, PI 89772, PI 90763 and PI 437654, revealing that these lines have same

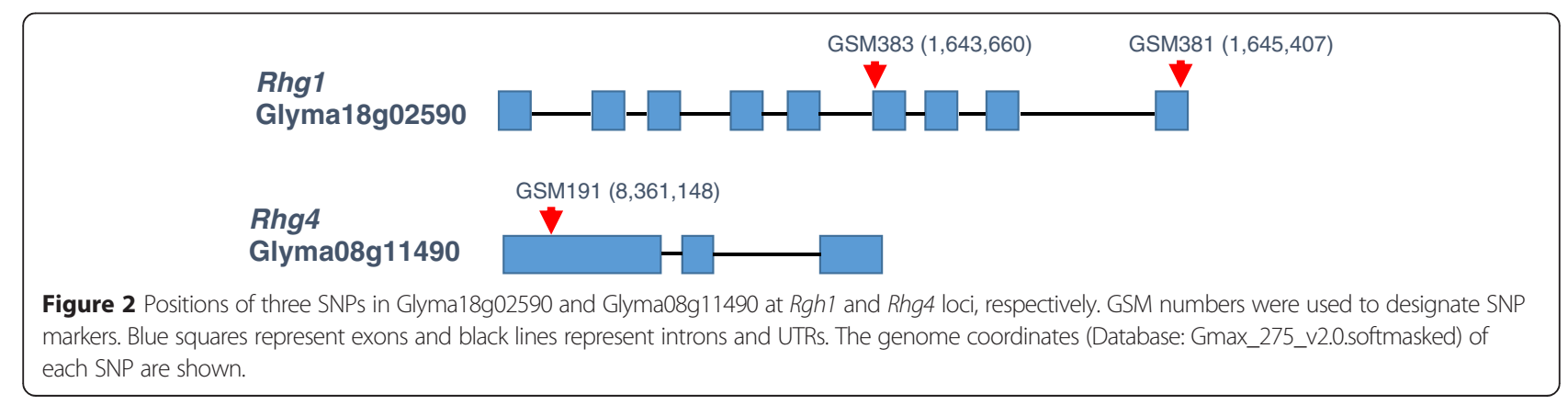


Table 1 Haplotype alleles at $R h g 1$ andRhg4 loci and source identification in 27 soybean lines ${ }^{*}$

\begin{tabular}{|c|c|c|c|c|c|}
\hline \multirow[t]{2}{*}{ Name } & \multicolumn{2}{|l|}{ Markers at Rhg1 locus } & \multirow{2}{*}{$\begin{array}{l}\text { Marker at Rhg4 locus } \\
\text { GSM191 (Gm08: } 8361148)\end{array}$} & \multirow{2}{*}{$\begin{array}{l}\text { Reaction to } \\
\text { SCN Race } 3\end{array}$} & \multirow{2}{*}{$\begin{array}{l}\text { Resistance } \\
\text { type }\end{array}$} \\
\hline & GSM381 (Gm18: 1645407) & GSM383 (Gm18: 1643660) & & & \\
\hline PI548655 (Forrest) & G & G & G & $\mathrm{R}$ & Peking \\
\hline PI548402 (Peking) & G & G & G & $\mathrm{R}$ & Peking \\
\hline Pl89772 & G & G & G & $\mathrm{R}$ & Peking \\
\hline PI90763 & G & G & G & $\mathrm{R}$ & Peking \\
\hline PI437654 & G & G & G & $\mathrm{R}$ & Peking \\
\hline PI548316 & G & C & C & $\mathrm{R}$ & PI 88788 \\
\hline Pl88788 & G & C & C & $\mathrm{R}$ & PI 88788 \\
\hline PI209332 & G & C & C & $\mathrm{R}$ & PI 88788 \\
\hline PI603372 & G & C & C & $\mathrm{R}$ & PI 88788 \\
\hline PI603587A & G & C & C & $\mathrm{R}$ & PI 88788 \\
\hline PI603384 & G & C & C & $\mathrm{S}$ & susceptible \\
\hline PI97094 & G & C & C & $S$ & susceptible \\
\hline PI548667 & $\mathrm{T}$ & C & C & $\mathrm{S}$ & susceptible \\
\hline PI518671 (Williams 82) & $\mathrm{T}$ & C & C & $S$ & susceptible \\
\hline PI567359 & $\mathrm{T}$ & C & $C$ & $S$ & susceptible \\
\hline PI567368 & $\mathrm{T}$ & C & C & $S$ & susceptible \\
\hline PI567481 & $\mathrm{T}$ & C & C & $S$ & susceptible \\
\hline Pl602991 & $\mathrm{T}$ & C & C & $S$ & susceptible \\
\hline Pl603357 & $\mathrm{T}$ & C & C & $\mathrm{S}$ & susceptible \\
\hline PI603420 & $\mathrm{T}$ & C & C & $S$ & susceptible \\
\hline PI603428C & $\mathrm{T}$ & C & C & $\mathrm{S}$ & susceptible \\
\hline PI603502A & $\mathrm{T}$ & C & C & $\mathrm{S}$ & susceptible \\
\hline Pl603656 & $\mathrm{T}$ & C & C & $S$ & susceptible \\
\hline PI567568B & $\mathrm{T}$ & C & C & $\mathrm{S}$ & susceptible \\
\hline PI587993 & $\mathrm{T}$ & C & C & $S$ & susceptible \\
\hline PI594770A & $\mathrm{T}$ & C & C & $\mathrm{S}$ & susceptible \\
\hline PI594773 & $\mathrm{T}$ & C & C & S & susceptible \\
\hline
\end{tabular}

${ }^{\ddagger}$ Lines were used by Cook et al. [12] and Liu et al. [14].

${ }^{\dagger}$ Numbers represent the genome coordinate based on the Database: Gmax_275_v2.0.softmasked.

haplotypes to Peking at the Rhg1 locus as large as 5.9 Mbp. A $788 \mathrm{~kb}$ haplotype window block was shown in Figure 3A. Hartwig, PI 89772, PI 90763 and PI 437654 showed a relatively small haplotype window $(710 \mathrm{~kb})$ when compared to Peking. Woodruff had a larger haplotype block that is about $3.8 \mathrm{Mbp}$ and over $5.9 \mathrm{Mbp}$ was observed in Forrest, Gordon and Bryan. However, PI 88788 and other susceptible-type lines showed no introgression from Peking at the Rhg1 locus. All eight PI 88788-type lines, G00-3880, Prichard, Bedford, Carver, PI 548316, PI 209332, PI 603372 and PI 603587A exhibited haplotype window blocks ranging from $1.6 \mathrm{Mbp}$ to $4.3 \mathrm{Mbp}$, when compared to PI 88788 at the Rhg1 region. Haplotype analysis of Rhg4 locus on Chr08 indicated haplotype window blocks from Peking and nine Peking-type lines (Figure 3B), with a smaller region of $348 \mathrm{~kb}$ in Woodruff, Boggs and Bryan. However, the introgressed haplotype blocks are as large as $2.6 \mathrm{Mbp}$ in Forrest, Hartwig and Gordon. Examination of the pedigrees of these lines indicated that Peking is present somewhere in the extended pedigree of these six cultivars (http://www.ars-grin.gov/npgs/acc/acc_ queries.html) [32-35]. These data are consistent with genotype indications of our three SNPs alleles and further substantiated the effectiveness of these SNP markers.

\section{Validation of SNP markers}

To validate the SNP markers with more diverse lines, we explored the data from the previous three years' USDA Uniform Soybean Tests for Southern States where the phenotypes of SCN race 3 are available for all the tested lines. We requested a total of 153 lines from eight institutions and performed KASP assays on these lines with SNP markers, GSM381, GSM383 and GSM191 (Figure 4A). The SNP genotypes, SCN race 3 reactions and 


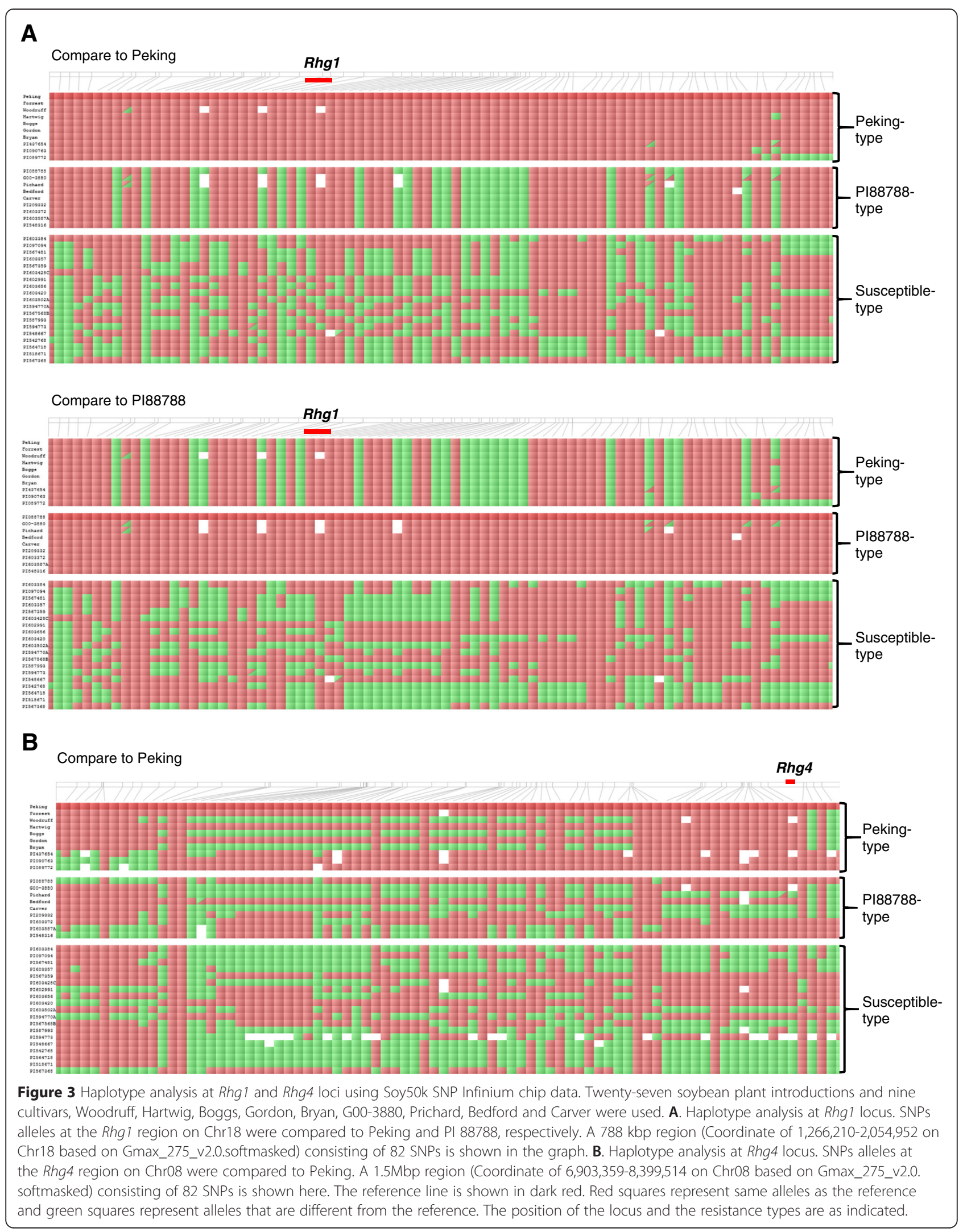




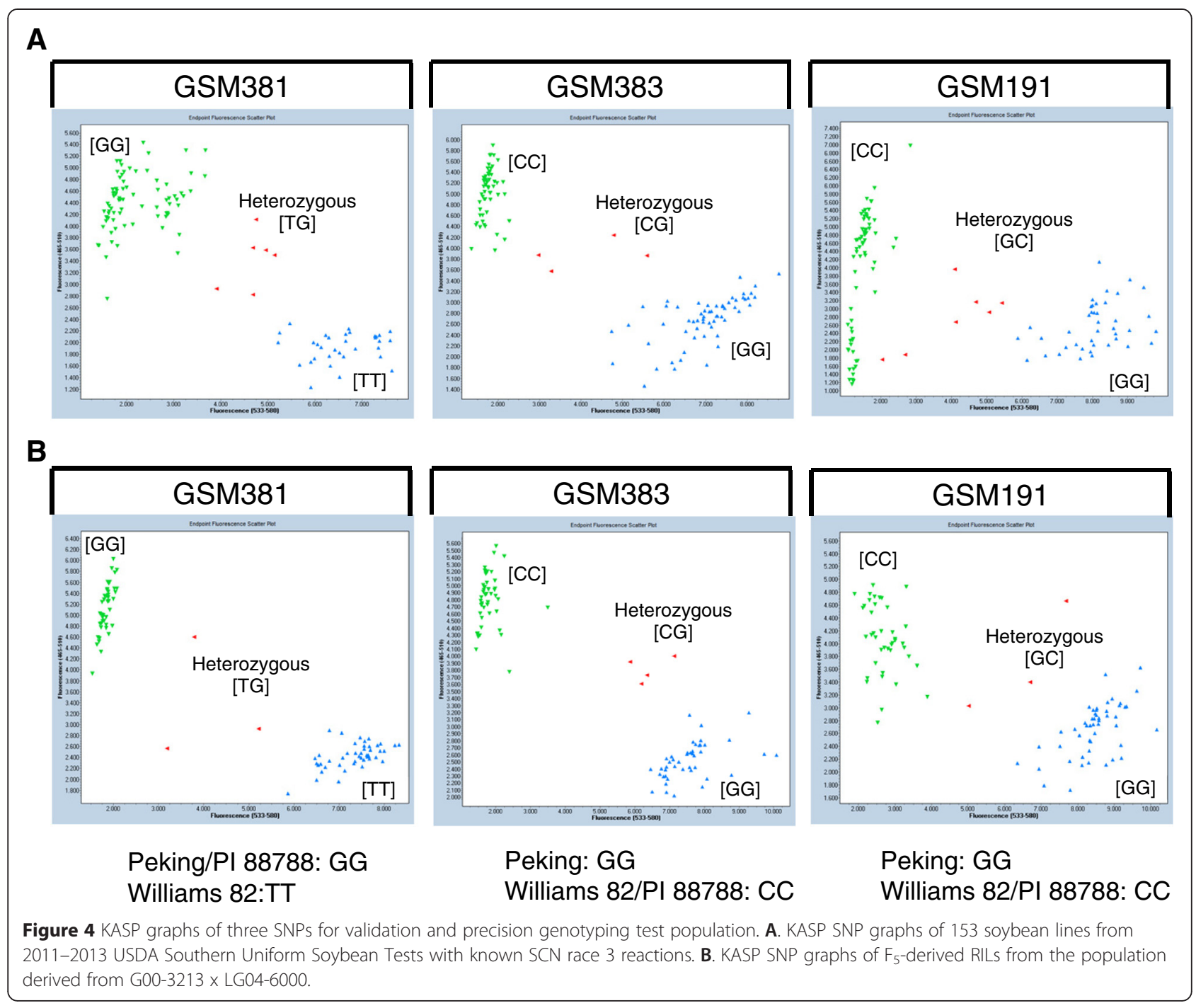

pedigrees were listed (Additional file 1: Table S1). The rating of SCN phenotype is on a scale of 1 to 5 based on the number of cysts, thus, lines with ratings smaller than 3 were considered as resistant and lines with ratings equal to or greater than 3 as susceptible. A strong accordance was observed between SNP genotypes and reactions to SCN race 3 for 145 germplasm lines with an exception of 10 lines (successful rate $>93 \%$ ). Due to the large discrepancy of ratings for same line between two years, eight lines were excluded for analysis. Single factor analysis using General Linear Model (GLM procedure, SAS 9.3) showed a significant correlation between SCN resistance phenotypes and genotypes consisted of three SNP markers $\left(\mathrm{R}^{2}=0.69, \mathrm{P}<\right.$ 0.001 ). Moreover, we have examined the extended pedigrees (data not shown) of these lines with resistance Rhg1 allele $(\mathrm{GSM} 381=\mathrm{G})$ to identify the sources of resistance (Additional file 1: Table S1). The SNP genotypes are in complete agreement with the resistance sources based on extended pedigrees: Peking-source with a $G$ allele at
GSM383 and PI 88788-source with a C allele, indicating that GSM383 is a functional SNP to differentiate Peking and PI 88788 sources for the Rhg1 resistance allele.

We also performed a precision genotyping test on a biparental population of $\mathrm{F}_{5}$-derived Recombinant inbred lines (RILs) from G00-3213 x LG04-6000. The line G00-3213 carries a Peking-source Rhg1 and Rhg4 resistance alleles $(\mathrm{GSM} 381=\mathrm{G}, \mathrm{GSM} 383=\mathrm{G}$, and $\mathrm{GSM} 191=\mathrm{G})$ and is resistant to SCN race 3, while LG04-6000 is a susceptible line with T, C and C alleles for GSM381, GSM383, and GSM191, respectively. Three replicates of 150 RILs with two parents were arranged in a randomized complete block design in greenhouse and each entry was genotyped with three SNP markers (Figure 4B). Ten weeks after inoculation, phenotyping was carried out by counting the numbers of cysts on the roots of each individual plant. Resulting phenotypes demonstrated that combination of the resistance Rhg1 $(\mathrm{GSM} 381=\mathrm{G}$ and $\mathrm{GSM} 383=\mathrm{G}$ ) and resistance Rhg4 alleles $(\mathrm{GSM} 191=\mathrm{G})$ brought a resistant phenotype with only 0.4 
cyst/plant and all the other combinations exhibited a more susceptible phenotype (Figure 5, Table 2), suggesting that these three markers can be employed for the selection of $\mathrm{SCN}$ race 3 resistance from a population derived from Peking source. Single factor analysis using a GLM procedure in SAS 9.3 was performed for three subsets individually and combined. All of them showed a significant correlation between the average number of cysts and genotypes of three SNPs, GSM381, GSM383, and GSM191 ( $\mathrm{P}<0.0001)$, with an R-square of $0.64,0.40,0.24$, and 0.3 for RIL sets $1,2,3$ and combined, respectively. The different $\mathrm{R}$-square values among sets might be due to the conditions at different inoculation times.

\section{Discussion}

The genetic basis of resistance to SCN is complex in soybean, not only because resistance to $\mathrm{SCN}$ is multigenic, but also because different sets of genes/ Quantitative trait loci (QTLs) confer resistance to the diverse populations of SCN due to their genetic heterogeneity. Field populations of soybean cyst nematode have been classified into 16 races based on their ability to develop on resistant cultivars [6]. Resistance to $\mathrm{SCN}$ is racecultivar specific [36], for example, soybean cultivars derived from Peking source are commonly claimed to be resistant to race 1 and 3 , however, the cultivars with SCN resistance from PI 88788 are usually shown to be resistant to race 3 and 14 [37,38]. A growing body of evidence has shown different defense loci that underlie resistance to different races. The Rhgl locus on Chr18 is necessary for resistance to all SCN races [39]. Many other cultivar-race specific QTLs have been demonstrated in resistance sources, such as $R h g 4$ for race 1 and 3, Rhg3 and Rhg 5 for race 2 and $R h g 2$ for race 5 and 14
Table 2 Genotypes of SNP markers and average number of cysts for $150 \mathrm{~F}_{5}$ families of G00-3213 x LG04-6000

\begin{tabular}{llll}
\hline SNP marker genotypes & GSM191 & $\begin{array}{l}\text { Average } \\
\text { numbers } \\
\text { of cysts }^{\dagger}\end{array}$ \\
\hline GG & GSM383 & CC & $11.1 \mathrm{~b}$ \\
$\mathrm{GG}$ & $\mathrm{GG}$ & $\mathrm{GG}$ & $0.40 \mathrm{a}$ \\
$\pi$ & $\mathrm{GG}$ & $\mathrm{CC}$ & $7.0 \mathrm{~b}$ \\
$\pi$ & $\mathrm{CC}$ & $\mathrm{GG}$ & $10.0 \mathrm{~b}$ \\
\hline
\end{tabular}

${ }^{\dagger}$ Means followed by a different letter are significantly different at a probability level of 0.01 .

$[15,38,40,41]$. Although two functional SNPs have been identified for Rhg4 locus [14], no high-throughput marker assays have been reported to date. Moreover, several SNPs have been found at Rhg1 locus [16], but no specific functional SNPs as well as detection assays have been reported to associate with the resistance allele, nor the SNPs to differentiate sources of resistance. Built on the previous discoveries of copy number variation, gene dosage, and DNA methylation at the Rhg1 locus by Cook et al. [16] and SNPs at the Rhg4 locus by Liu et al. [14], in this study, we have developed SNP marker assays, GSM381, GSM383, and GSM191 at Rhg1 and Rhg4 locus for the selection of resistance alleles, (Figure 2). The SNPs reside in the coding sequences of resistance genes (Glyma18g02590 and Glyma08g11490) and also introduce changes in protein sequences, thus they can be considered as functional markers for SCN race 3 resistance and applied in high throughput marker-assisted selection with high accuracy. Similarly, the knowledge of additional loci along with the genome sequence of soybean may be used in the marker development for resistance to different $\mathrm{SCN}$ races.

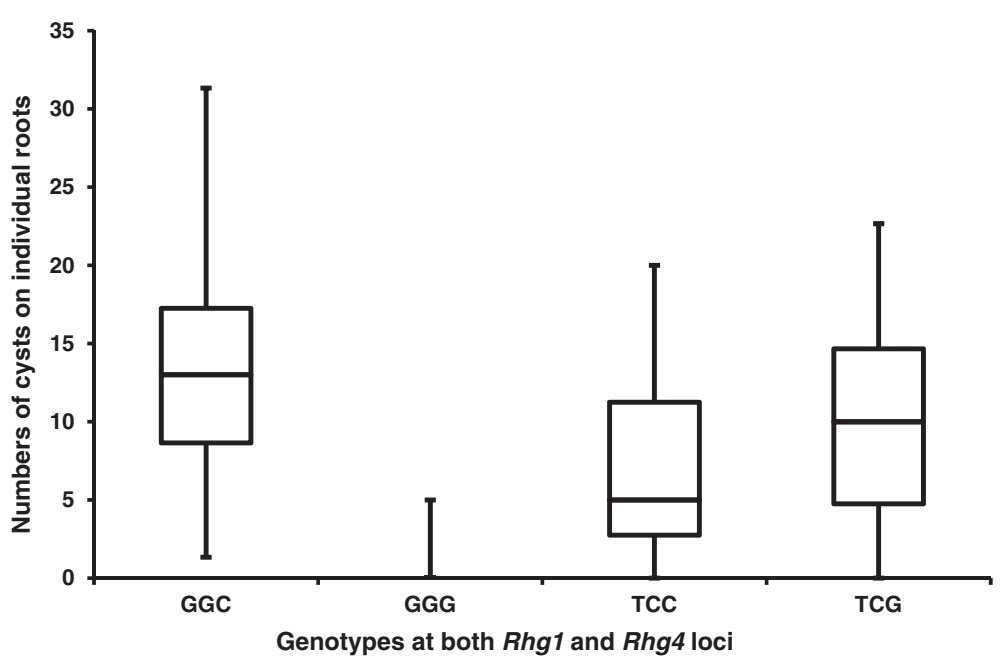

Figure 5 Reactions of race 3 to different combinations of SNP marker alleles for 150 F families of G00-3213 x LG04-6000. Rhg 1 and Rhg 4 genotypes are represented as combinations of alleles of SNP markers, GSM381, GSM383 and GSM191. Boxes show median, 25\% to 75\% of the data and whiskers extend to minimum and maximum of the data. 
PI 88788 has been the primary choice in soybean cultivar development as a source for SCN resistance [1], however, SCN populations have demonstrated the capability to mutate and recombine to new races, which can overcome the soybean resistance derived from PI 88788 [36]. Reports have shown that PI 88788 has not been providing sufficient protection against SCN in some fields [42-45]. Thus, additional resistance sources are desired to be used for developing new resistant cultivars and Peking is believed to be an attractive option. Although SNP GSM381 discriminates resistant and susceptible $R h g 1$ alleles, it does not distinguish PI 88788- and Peking-type Rhg1 loci. Thus, to identify the sources of resistance, we demonstrated that one SNP marker at Rhg1 locus, GSM383 was able to distinguish Peking from PI 88788 loci for most soybean germplasm (Figure 4 and Additional file 1: Table S1). This SNP can be employed in differentiating Peking from PI 88788 sources for the Rhg1 allele for breeding programs.

The successful prediction rate for three SNP markers at Rhg1 and Rhg4 loci is over 93\% in this study (Figure 4 and Additional file 1: Table S1). A few lines (10 lines from USDA Uniform Test (Additional file 1: Table S1) and two lines from 27 validation lines (Table 1)) did not show a correlation between the expected phenotypes and the resistance SNP alleles. A number of factors may result in the discrepancy between genotyping and phenotyping results, including 1) phenotyping/ or seed source errors: some of the lines have been tested for only one year, and one-time phenotyping of SCN resistance may not be definitive; 2) sources other than PI 88788 and Peking may be providing resistance; and 3) the complexity of the $R h g 1$ region is not fully understood. A future study will be designed to understand why the lines with the resistance genotypes did not have a resistance reaction to $\mathrm{SCN}$ race 3 . The $31.2 \mathrm{~kb}$ region at $\operatorname{Rgh} 1$ locus is repeated in resistant genotypes [12], and the sequences of the individual copies are also variant within a single genotype, which makes marker development challenging for the Rhg1 locus. We also tested marker performance with artificial heterozygous DNA, where PI 88788/ Peking and Williams 82 DNA were mixed at 1:1 ratio. Although the artificial heterozygous allele cluster was located a little closer towards the resistant homozygous allele cluster in the KASP SNP graphs (data not shown) because of the gene repeats in resistant lines, the alleles can still be called clearly. Therefore, the functional SNP markers that we identified can be used not only to select the resistance allele, but also to differentiate resistance sources, which were also confirmed by analysis of the Rhg1 region with Soy50k SNP Infinium chip data using 38 soybean lines (Figure 3A).

A recent study of the Rhg1 locus revealed that copy number variation of the $31 \mathrm{~kb}$ DNA fragment, DNA sequence variation, and differentially methylated patterns were correlated with the Rhg1-mediated resistance to $\operatorname{SCN}[12,16]$. These discoveries may also offer additional selection strategies for the Rhg1 allele, in addition to the functional SNP detection assays we developed. Cook et al. [16] explored the whole genome data for Rhg1 locus in 42 soybean lines, and multiple lines are in common in both studies. In this study, we got the same C-terminal protein sequence of Glyma18g02590 for both low-copy and high-copy Rhg1 as described in the previous study ([16] Figure 3B), indicating that GSM 381 maybe also exist in their DNA sequences. Interestingly, they identified a novel splice isoform of Glyma18g02590 cDNA in Peking-type Rhg1 lines, however, we did not detect this isoform in our cDNA sequencing, probably due to the low copy number of this isoform and use of a different sequencing method. Thus, more studies are needed to investigate the Rhg1 locus in various soybean lines for new insights into the development of improved selection approaches.

Soybean cyst nematode is estimated to cause more than one billion dollars yield loss annually in the USA [2], and thus nematode resistance is of tremendous interest to soybean breeders and growers. The SNP markers we identified could be used to guide the screening of soybean germplasm for SCN resistance for both Peking and PI 88788 sources, as well as to improve the efficiency and precision of selection for SCN resistance in breeding programs. If the known resistance source was used as one of the parents in a cross, only GSM381 is needed to select for PI 88788-type resistance, while both GSM383 and GSM191 are required to select for Peking-type resistance. Moreover, the KASP assays that we developed were PCR-based assays and robust in detecting the resistance allele and separating heterozygotes from homozygotes and thus can be used in highthroughput selection of SCN resistance. Finally, with aid of molecular markers, multiple SCN resistance genes can be pyramided into elite soybean genotypes in a more timely and labor-efficient manner.

\section{Conclusions}

The genomic sequence of Glyma08g11490 at Rhg4 locus in 28 soybean lines and Glyma18g02590 at Rhg1 locus in 11 lines, along with the known reactions to soybean cyst nematode race 3 of these lines, allowed us to identify functional SNPs and develop robust KASP SNP assays for marker-assisted breeding. Using a panel of 153 soybean germplasm and a segregating bi-parent population, we have validated the usefulness of these three SNP markers, two at Rhg1 locus and one at Rhg4. The results of this work demonstrated that these markers could be applied for high throughput selection of SCN resistance from PI 88788 and Peking sources and for accelerating breeding of $\mathrm{SCN}$ resistance in an accurate and efficient manner. 


\section{Methods}

\section{Plant materials}

Twenty-seven soybean lines with known SCN phenotypes that were used in both studies by Cook et al. [12] and Liu et al.[14] (Listed in Table 1) were selected for the initial sequencing and SNP identification. The seeds were obtained from the USDA Soybean Germplasm Collection (Urbana, IL) and grown in the greenhouse. Leaf tissue was collected from 15 plants of each line for bulk DNA extraction.

One hundred and fifty-three soybean lines that were entered into the USDA Uniform Soybean Tests for Southern States from 2011 to 2013 with known SCN reactions were selected for SNP marker validation. These lines were kindly provided by their respective breeders. The seed was planted in 32 oz cups in the greenhouse with 15 seed/line. Twelve leaves were pooled from 12 plants with one from each plant and samples were freeze-dried for 48 hours to be used for DNA extraction.

The $150 \mathrm{~F}_{5: 6}$ RIL lines were derived from the cross of G00-3213 x LG04-6000 using a single seed descent method. G00-3213 is a high-yielding line with nematode resistance developed at the University of Georgia. LG04-6000 (Reg. No. GP-379, PI 664025) is known to be genetically diverse and high-yielding line that was developed and released by the USDA-Agricultural Research Service and the Illinois Agricultural Experiment Station, Urbana IL.

\section{Sequencing and SNP identification}

To discover SNPs at the Rhg1 locus, the genomic sequences of three genes, Gm18g02580, Gm18g02590 and Gm18g02610 from Williams 82 were obtained from Phytozome (http://www.phytozome.net/). The $31.2 \mathrm{~kb}$ region from PI 88788 and the cDNA sequence of Gm18g02590 were acquired from NCBI (JX907806) and the publication of Matsye et al. [31], respectively. DNA alignment was carried out using Geneious version 5.5.7 and potential SNPs were identified. To obtain additional SNPs at the Rhgl locus, genomic DNA and cDNA of Gm18g02590 was sequenced from 11 soybean lines (Forrest, PI 437654, Peking, PI 89772, PI 90763, PI 88788, PI 548316, PI 209332, Williams 82, PI 603428C and Essex) and four lines (Forrest, Essex, Williams 82 and PI 88788) with known phenotype reactions, respectively. Four pairs of specific primers (Additional file 2: Table S2) were designed according to Williams 82 genomic sequence of Gm18g02590. At least two independent PCR amplifications were performed for each primer pair. Individual PCR products were cloned into the pGEM-T vector and 10 clones were randomly selected for each PCR amplification and sequenced. The entire gene sequences were obtained from the alignment of the corresponding four fragments and blasted with Williams 82 genomic sequence.
To identify the SNPs at the Rhg4 locus, the genomic DNA of Gm08g11490 was sequenced from 28 lines (above 27 lines and RESSEQ). The collection of soybean lines used in sequencing was obtained from the USDA Soybean Germplasm Collection (Urbana, IL). The soybean seed was planted in the greenhouse of the Horticultural Research Center at Southern Illinois University, Carbondale, IL. Young leaf tissue of the soybean lines was harvested to extract DNA using the DNeasy Plant Mini Kit (Qiagen). According to the genome reference sequence of Williams 82 , the specific primers were designed to amplify the genomic DNA fragments of GmSHMT and GmSNAP with a 38 cycles of PCR amplification at $94^{\circ} \mathrm{C}$ for $30 \mathrm{~s}, 50-60^{\circ} \mathrm{C}$ for $30 \mathrm{~s}$ and $72^{\circ} \mathrm{C}$ for $90 \mathrm{~s}$. The PCR products were purified using the QIAquick PCR Purification Kit (Qiagen). Then, the purified PCR fragments were sequenced at GENEWIZ Company (www.genewiz.com), and the sequences were aligned. Primers used in sequencing were listed in Additional file 2: Table S2.

\section{Haplotype analysis}

To understand the genetic variation at both the Rhg1 and Rhg4 loci, Soy50K SNP Infinium chip data for 27 above soybean lines and nine cultivars, Woodruff, Hartwig, Boggs, Gordon, Bryan, G00-3880, Prichard, Bedford and Carver were obtained from Soybase (www.soybase.org) or our laboratory database at the University of Georgia, respectively. The SNPs on Chr18 and Chr08 for these 36 lines were imported into Flapjack software [46] along with the corresponding marker genetic map file for visualization of the haplotypes around Rhg1 and Rhg4 loci. The color scheme of "By similarity to line" was applied to compare to SNP alleles of Peking and PI 88788 and the region of introgression from sources was determined by the haplotype markers with different alleles to the reference lines.

\section{KASP assays design and SNP validation}

To test the association of SNPs with the reactions to $\mathrm{SCN}$ race 3 and develop robust markers for high throughput selection, Kompetitive Allele Specific PCR (KASP) assays were developed and tested for all the identified SNPs using above 27 soybean lines with known SCN phenotypes. The assays were performed as previously described [47]. Three SNPs, GSM381 (in Glyma18g02590, G/T), GSM383 (in Glyma18g02590, G/C) and GSM191 (in Glyma08g11490, G/C), were then selected for marker validation. Primer sequences are listed in Table 3 . KASP assays were run with $4 \mu \mathrm{L}$ reaction system including $2 \mu \mathrm{L}$ low rox KASP master mix (KBiosciences, Herts England), $0.106 \mu \mathrm{L}$ of primer mix $(0.318 \mu \mathrm{L}$ of each primer at final concentration) and $2 \mu \mathrm{L}$ of $10-25 \mathrm{ng} / \mu \mathrm{l}$ genomic DNA. The PCR conditions for KASP marker assay was $94^{\circ} \mathrm{C}$ for $15 \mathrm{~min}$, followed by 10 cycles of touch down PCR from $68^{\circ} \mathrm{C}$ to $60^{\circ} \mathrm{C}$ with 
Table 3 KASP assay primer sequences of three SNPs, GSM381, GSM383 and GSM191

\begin{tabular}{|c|c|c|c|}
\hline Locus & Gene & Marker & \\
\hline \multirow[t]{3}{*}{ Rhgl } & Gm18g02590 & GSM381 & FAM_primer: GAAGGTGACCAAGTTCATGCTAGCCAAAGAACTTGAGSAGBATGAG \\
\hline & & & VIC_Primer: GAAGGTCGGAGTCAACGGATTAGCCAAAGAACTTGAGSAGBATGAT \\
\hline & & & Common reverse primer: CAAACAATAGGTCCAACCACCA \\
\hline \multirow[t]{3}{*}{ Rhg1 } & Gm18g02590 & GSM383 & FAM_primer: GAAGGTGACCAAGTTCATGCTATCTGCMAACTCTGTAAAGAGGAC \\
\hline & & & VIC_Primer: GAAGGTCGGAGTCAACGGATTATCTGCMAACTCTGTAAAGAGGAG \\
\hline & & & Common reverse primer: GCTGTCCAGTCTCCAGAAGTGAA \\
\hline \multirow[t]{3}{*}{ Rhg4 } & Gm08g11490 & GSM191 & FAM_primer: GAAGGTGACCAAGTTCATGCTCATCATGGGGCTAGATCTCCC \\
\hline & & & VIC_Primer: GAAGGTCGGAGTCAACGGATTCATCATGGGGCTAGATCTCCG \\
\hline & & & Common reverse primer: TAGCCGGTGGTGGAGTTACC \\
\hline
\end{tabular}

$0.8^{\circ} \mathrm{C}$ decrease per cycle, then followed by 30 cycles of $94^{\circ} \mathrm{C}$ for $20 \mathrm{~s}$ and $57^{\circ} \mathrm{C}$ for $1 \mathrm{~min}$. . PCR fluorescent endpoint readings were performed using the Light $\mathrm{Cycler}^{\oplus}$ 480 Real-Time PCR System (Roche, Germany).

The seeds of 153 soybean lines were planted in $32 \mathrm{oz}$ cups in the greenhouse with 15 seeds per line. One leaf from each of 12 plants were pooled and leaf samples were freeze-dried for 48 hours. DNA was isolated using a 96well plates as described in [48]. KASP assays were performed for three SNP markers with Peking (resistant) and Williams 82 (susceptible) as controls. Associations of the markers with SCN resistance were tested using singlefactor analysis of variance with a General Linear Model (GLM) procedure in SAS 9.3 (SAS Institute, 2013) based on the known phenotype reactions to $\mathrm{SCN}$ race 3 from USDA Uniform Soybean Tests for Southern States.

The $150 \mathrm{~F}_{5}$-derived recombinant inbred lines (RILs) of G00-3213 x LG04-6000 were randomized and grown in the greenhouse along with the parents and controls for phenotyping and tissue sampling for DNA extraction. DNA extraction procedures and KASP assays for three SNP markers were performed as described above. Associations of the markers with SCN resistance were tested using single-factor analysis of variance with a GLM procedure in SAS 9.3 (SAS Institute, 2013) based on means of the cyst numbers from three replicates for each subset separately and combined.

\section{SCN phenotyping assays}

The phenotypic data for reactions to SCN race 3 and direct pedigrees of soybean lines were acquired from 2011-2013 USDA Uniform Soybean Tests for Southern States [49-51]. The extended pedigrees were acquired by searching the pedigree of immediate parents on GRIN and/or past USDA Uniform Soybean Tests for Southern States. The screenings for SCN reactions were conducted in the USDA-ASR-SEA greenhouse, Jackson, TN. One seed of each line was planted in sterile soil mix in a three inch sterile clay pot. Screening for SCN was done with HG type 0 (race 3 ) in 2011, HG type 5.7 (race 3 ) in
2012 and HG type 5.7 (race 3) in 2013. Three replications were used for each entry per HG Type. At the time of planting, approximately 2,000 eggs of the nematode population (HG Type) extracted from crushed SCN females were added to each pot. Thirty days (+/ -1 day) after planting, plants were rated based on the number of cysts on the roots. The rating scale was as follows: $1=0$ 5 cysts; $2=6-10$ cysts; $3=11-20$ cysts; $4=21-40$ cysts; and $5=>40$ cysts [49]. Homogeneous nematode populations that had been cultured in the greenhouse for several reproductive cycles were used. The HG Type was confirmed using established indicator lines [7].

The $150 \mathrm{~F}_{5}$-derived RILs of G00-3213 x LG04-6000 were divided into three subsets for greenhouse phenotyping, each with 50 lines and parents. Parental lines G003213 and LG04-6000, and resistant and susceptible controls, Bryan [34] and Haskell [52], were also included twice in each set. Three replications for each line were arranged in a randomized complete block design and three seeds for each replication were planted into a $2.5 \mathrm{~cm}$-diameter cone-tainer. One week after planting, the plants were thinned to one and the roots were inoculated with 4,000 SCN race 3 eggs using a digital dispensing pump. Ten weeks after inoculation, the numbers of cysts were counted on each individual plant.

\section{Additional files}

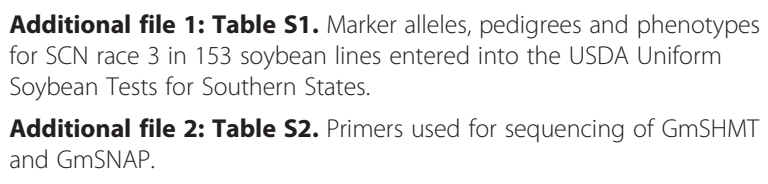

\section{Abbreviations}

SCN: Soybean cyst nematode; SSR: Simple sequence repeat; Rhg: Resistance to H. glycines; Chr: Chromosome; SNP: Single nucleotide polymorphism;

InDel: Insertion/ deletion; KASP: Kompetitive Allele Specific; PCR: Polymerase chain reaction; HG: Heterodera glycines; RIL: Recombinant inbred lines; GLM: General linear model; RFLP: Restriction fragment length polymorphism; SHMT: Serine hydroxymethyltransferase; UTR: Untranslated region; QTL: Quantitative trait loci. 


\section{Competing interests}

The authors declare that they have no competing interests.

\section{Authors' contributions}

$\mathrm{ZS}$ and $\mathrm{ZL}$ contributed to designing and conducting the experiment. ZS was involved in implementation of most experimental plans and data acquisition. $\mathrm{SL}$ and KM sequenced genomic DNA at both loci and performed the sequence analysis. JN contributed to the greenhouse SCN phenotyping. PA was involved in SCN phenotyping of soybean lines in USDA Uniform Tests. $\mathrm{ZS}$ and $\mathrm{ZL}$ wrote the manuscript with edits from JN and other authors. All authors read and approved the final manuscript.

\section{Acknowledgements}

We would like to acknowledge the following people for providing the seeds of germplasm: Dr. Grover Shannon, University of Missouri; Dr. Pengyin Chen, University of Arkansas; Dr. Thomas E. Carter, USDA and North Carolina State University; Drs. Emerson Shipe and Ben Fallen, Clemson University; Dr. Vince Pantalone, University of Tennessee; Dr. Bo Zhang, Virginia Tech. Thanks to Dr. H. Roger Boerma at the University of Georgia for developing G00-3213 x LG04-6000 population, and Dale Wood and Steve Finnerty for their technical assistance in planting preparation and SCN phenotyping in greenhouse. We also thank Joao Cenci, Colleen Wu and Tatyana Nienow for their assistance with sampling and genotyping.

This research was supported by the funding from the United Soybean Board and the University of Georgia Research Foundation to ZL, ZS, and JN; by the funding from the United Soybean Board and the Illinois Soybean Association to KM and SL; and by the funding from the United Soybean Board and the Tennessee Soybean Promotion Board to PA. The funding agencies had no role in study design, data collection and analysis, interpretation of data, decision to publish, or preparation of the manuscript.

\section{Author details}

${ }^{1}$ Center for Applied Genetic Technologies \& Dep. of Crop and Soil Sciences, University of Georgia, Athens, GA 30602, USA. ${ }^{2}$ Department of Plant, Soil and Agriculture Systems, Southern Illinois University, Carbondale, IL 62901, USA. ${ }^{3}$ Department of Plant Pathology, University of Georgia, Athens, GA 30602, USA. ${ }^{4}$ USDA-ARS-SEA, Jackson, TN 38301, USA.

\section{Received: 25 November 2014 Accepted: 13 April 2015 Published online: 18 April 2015}

\section{References}

1. Niblack TL, Lambert KN, Tylka GL. A model plant pathogen from the kingdom animalia: Heterodera glycines, the soybean cyst nematode. Annu Rev Phytopathol. 2006;44:283-303.

2. Koenning SR, Wrather JA. Suppression of Soybean Yield Potential in the Continental United States by Plant Diseases from 2006 to 2009 Plant Health Progress. 2010. doi:10.1094/PHP-2010-1122-01-RS.

3. Concibido VC, Diers BW, Arelli PR. A decade of QTL mapping for cyst nematode resistance in soybean. Crop Sci. 2004;44(4):1121-31.

4. Ferreira MFD, Cervigni GDL, Ferreira A, Schuster I, Santana FA, Pereira WD, et al. QTLs for resistance to soybean cyst nematode, races 3, 9, and 14 in cultivar Hartwig. Pesqui Agropecu Bras. 2011;46(4):420-8.

5. Guo B, Sleper DA, Nguyen HT, Arelli PR, Shannon JG. Quantitative trait loci underlying resistance to three soybean cyst nematode populations in soybean PI 404198A. Crop Sci. 2006;46(1):224-33.

6. Riggs RD, Schmitt DP. Complete Characterization of the Race Scheme for Heterodera-Glycines. J Nematol. 1988;20(3):392-5.

7. Niblack TL, Arelli PR, Noel GR, Opperman CH, Ore JH, Schmitt DP, et al. A revised classification scheme for genetically diverse populations of Heterodera glycines. J Nematol. 2002;34(4):279-88.

8. Jackson G. Soybean Cyst Nematode. 2012. http://msucares.com/pubs/ publications/p1293.pdf. Accessed 15 Apr 2015.

9. Mulrooney RP. Soybean Cyst nematode. 2011. http://extension.udel.edu/ factsheet/soybean-cyst-nematode/. Accessed 15 Apr 2015.

10. Concibido VC, Lange DA, Denny RL, Orf JH, Young ND. Genome mapping of soybean cyst nematode resistance genes in 'Peking', PI 90763, and PI 88788 using DNA markers. Crop Sci. 1997;37(1):258-64.

11. Li YH, Zhang C, Gao ZS, Smulders MJM, Ma ZL, Liu ZX, et al. Development of SNP markers and haplotype analysis of the candidate gene for rhg1, which confers resistance to soybean cyst nematode in soybean. Mol Breeding. 2009;24(1):63-76.

12. Cook DE, Lee TG, Guo XL, Melito S, Wang K, Bayless AM, et al. Copy Number Variation of Multiple Genes at Rhg1 Mediates Nematode Resistance in Soybean. Science. 2012;338(6111):1206-9.

13. Diers BW, Arelli P. Management of parasitic nematodes of soybean through genetic resistance. In: 1999: Proceedings of the World Soybean Research Conference, 6th; Aug. 4-7 1999; Chicago, IL. Chicago, IL: Superior Printing; 1999. p. 1300-6.

14. Liu S, Kandoth PK, Warren SD, Yeckel G, Heinz R, Alden J, et al. A soybean cyst nematode resistance gene points to a new mechanism of plant resistance to pathogens. Nature. 2012;492(7428):256-60.

15. Meksem K, Pantazopoulos P, Njiti VN, Hyten LD, Arelli PR, Lightfoot DA. 'Forrest' resistance to the soybean cyst nematode is bigenic: saturation mapping of the Rhg1 and Rhg4 loci. Theor Appl Genet. 2001;103(5):710-7.

16. Cook DE, Bayless AM, Wang K, Guo XL, Song QJ, Jiang JM, et al. Distinct Copy Number, Coding Sequence, and Locus Methylation Patterns Underlie Rhg1-Mediated Soybean Resistance to Soybean Cyst Nematode. Plant Physiol. 2014;165(2):630-47.

17. Arelli AP, Anand SC, Wrather JA. Soybean Resistance to Soybean Cyst Nematode Race-3 Is Conditioned by an Additional Dominant Gene. Crop Sci. 1992;32(4):862-4

18. Concibido VC, Denny RL, Boutin SR, Hautea R, Orf JH, Young ND. DNA Marker Analysis of Loci Underlying Resistance to Soybean Cyst-Nematode (Heterodera-Glycines Ichinohe). Crop Sci. 1994;34(1):240-6.

19. Qiu BX, Arelli PR, Sleper DA. RFLP markers associated with soybean cyst nematode resistance and seed composition in a 'Peking' $x$ 'Essex' population. Theor Appl Genet. 1999;98(3-4):356-64.

20. Vierling RA, Faghihi J, Ferris VR, Ferris JM. Association of RFLP markers with loci conferring broad-based resistance to the soybean cyst nematode (Heterodera glycines). Theor Appl Genet. 1996;92(1):83-6.

21. Suzuki C, Tanaka Y, Takeuchi T, Yumoto S, Shirai S. Genetic relationships of soybean cyst nematode resistance originated in Gedenshirazu and PI84751 on Rhg1 and Rhg4 loci. Breeding Sci. 2012;61(5):602-7.

22. Cregan PB, Mudge J, Fickus EW, Danesh D, Denny R, Young ND. Two simple sequence repeat markers to select for soybean cyst nematode resistance coditioned by the rhg1 locus. Theor Appl Genet. 1999;99(5):811-8.

23. Weisemann JM, Matthews BF, Devine TE. Molecular Markers Located Proximal to the Soybean Cyst Nematode Resistance Gene, Rhg4. Theor Appl Genet. 1992;85(2-3):136-8.

24. Mudge J, Cregan PB, Kenworthy JP, Kenworthy WJ, Orf JH, Young ND. Two microsatellite markers that flank the major soybean cyst nematode resistance locus. Crop Sci. 1997;37(5):1611-5.

25. Chen YW, Wang DC, Arelli P, Ebrahimi M, Nelson RL. Molecular marker diversity of SCN-resistant sources in soybean. Genome. 2006;49(8):938-49.

26. Carter TE, Koenning SR, Burton JW, Rzewnicki PE, Villagarcia MR, Bowman DT, et al. Registration of 'N7003CN' Maturity-Group-VII Soybean with High Yield and Resistance to Race 2 (HG Type 1.2.5.7-) Soybean Cyst Nematode. J Plant Regist. 2011;5(3):309-17.

27. Arelli PR, Pantalone VR, Allen FL, Mengistu A. Registration of Soybean Germplasm JTN-5303. J Plant Regist. 2007;1(1):69-70.

28. Meksem K, Ruben E, Hyten DL, Schmidt ME, Lightfoot DA. High-throughput genotyping for a polymorphism linked to soybean cyst nematode resistance gene Rhg4 by using Taqman (TM) probes. Mol Breeding. 2001;7(1):63-71

29. Huabang Chen JF, Ferris V, Hogue P, Miller K, Vierling R. A Single Nucleotide Polymorphism Linked to the Quantitative Trait Locus of Soybean Cyst Nematode Resistance. Seed Technology. 2005;27(2):269-72.

30. Li YH, Reif JC, Jackson SA, Ma YS, Chang RZ, Qiu LJ. Detecting SNPS underlying domestication-related traits in soybean. BMC Plant Biol. 2014;14(1):251.

31. Matsye PD, Lawrence GW, Youssef RM, Kim KH, Lawrence KS, Matthews BF, et al. The expression of a naturally occurring, truncated allele of an alpha-SNAP gene suppresses plant parasitic nematode infection. Plant Mol Biol. 2012;80(2):131-55.

32. Boerma HR, Hussey RS, Phillips DV, Wood ED, Rowan GB, Finnerty SL. Registration of 'Boggs' soybean. Crop Sci. 2000;40(1):294-5.

33. Anand SC. Registration of Hartwig Soybean. Crop Sci. 1992;32(4):1069-70.

34. Boerma HR, Hussey RS, Phillips DV, Wood ED, Finnerty SL. Registration of Bryan Soybean. Crop Sci. 1991;31(2):487.

35. Boerma HR, Hussey RS, Wood ED, Barrett GB, Finnerty SL. Registration of Gordon Soybean. Crop Sci. 1985;25(4):711-2. 
36. Niblack TLNG, Lambert KL. The Illinois SCN type test: practical application of the HG Type classification system. J Nematol. 2003;35:345-55.

37. Skorupska HT, Choi IS, Raoarelli AP, Bridges WC. Resistance to Soybean Cyst-Nematode and Molecular Polymorphism in Various Sources of Peking Soybean. Euphytica. 1994;75(1-2):63-70.

38. Glover KD, Wang D, Arelli PR, Carlson SR, Cianzio SR, Diers BW. Near isogenic lines confirm a soybean cyst nematode resistance gene from PI 88788 on linkage group. J Crop Sci. 2004;44(3):936-41.

39. Kazi S, Shultz J, Afzal J, Hashmi R, Jasim M, Bond J, et al. Iso-lines and inbred-lines confirmed loci that underlie resistance from cultivar 'Hartwig' to three soybean cyst nematode populations. Theor Appl Genet. 2010;120(3):633-44.

40. Webb DM, Baltazar BM, Raoarelli AP, Schupp J, Clayton K, Keim P, et al. Genetic-Mapping of Soybean Cyst-Nematode Race-3 Resistance Loci in the Soybean Pi-437.654. Theor Appl Genet. 1995;91(4):574-81.

41. Schuster I, Abdelnoor RV, Marin SRR, Carvalho VP, Kiihl RAS, Silva JFV, et al. Identification of a new major QTL associated with resistance to soybean cyst nematode (Heterodera glycines). Theor Appl Genet. 2001;102(1):91-6.

42. Delheimer JC, Niblack T, Schmidt M, Shannon G, Diers BW. Comparison of the Effects in Field Tests of Soybean Cyst Nematode Resistance Genes from Different Resistance Sources. Crop Sci. 2010;50(6):2231-9.

43. Niblack T, Colgrove A, Colgrove K, Bond J. Shift in Virulence of Soybean Cyst Nematode is Associated with Use of Resistance from PI 88788. Plant Health Progress. 2008. doi:10.1094/PHP-2008-0118-1001-RS.

44. Chen S, Potter B, Orf J. Virulence of the Soybean Cyst Nematode Has Increased over Years in Minnesota. J Nematol. 2010;42(3):238.

45. Mulrooney RP, Gregory NF, Heinz RD. Soybean Cyst Nematode Populations in Delaware Are Shifting in Response to Widespread Planting of Soybean Cultivars with Resistance from Pi88788. J Nematol. 2010;42(3):259-60.

46. Milne I, Shaw P, Stephen G, Bayer M, Cardle L, Thomas WTB, et al. Flapjackgraphical genotype visualization. Bioinformatics. 2010;26(24):3133-4.

47. Pham AT, McNally K, Abdel-Haleem H, Boerma HR, Li ZL. Fine mapping and identification of candidate genes controlling the resistance to southern root-knot nematode in PI 96354. Theor Appl Genet. 2013;126(7):1825-38.

48. Keim P, Olson TC, Shoemaker RC. A rapid protocol for isolating soybean DNA. Soybean Genet Newsletter. 1988;15:150-2.

49. Gillen AM, Shelton GW. Uniform Soybean Test Southern States. 2013. http://www.ars.usda.gov/SP2UserFiles/Place/60661000/ UniformSoybeanTests/2013SoyBook.pdf). Accessed 15 Apr 2015.

50. Gillen AM, Shelton GW. Uniform Soybean Test Southern States. 2011. http://www.ars.usda.gov/SP2UserFiles/Place/60661000/ UniformSoybeanTests/2011SoyBook.pdf. Accessed 15 Apr 2015.

51. Gillen AM, Shelton GW. Uniform Soybean Test Southern States. 2012 http://www.ars.usda.gov/SP2UserFiles/Place/60661000/ UniformSoybeanTests/2012SoyBook.pdf. Accessed 15 Apr 2015.

52. Boerma HR, Hussey RS, Phillips DV, Wood ED, Finnerty SL. Registration of Haskell Soybean. Crop Sci. 1994;34(2):541.

\section{Submit your next manuscript to BioMed Central and take full advantage of:}

- Convenient online submission

- Thorough peer review

- No space constraints or color figure charges

- Immediate publication on acceptance

- Inclusion in PubMed, CAS, Scopus and Google Scholar

- Research which is freely available for redistribution 